\title{
Strategies for optimization of hexahedral meshes and their comparative study
}

\author{
J. I. López ${ }^{1}$ (1) M. Brovka ${ }^{1}$ J. M. Escobar ${ }^{1}$ R. Montenegro ${ }^{1}$ - G. V. Socorro ${ }^{1}$
}

Received: 2 January 2016 / Accepted: 29 April 2016

(C) Springer-Verlag London 2016

\begin{abstract}
In this work, we study several strategies based on different objective functions for optimization of hexahedral meshes. We consider two approaches to construct objective functions. The first one is based on the decomposition of a hexahedron into tetrahedra. The second one is derived from the Jacobian matrix of the trilinear mapping between the reference and physical hexahedral element. A detailed description of all proposed strategies is given in the present work. Some computational experiments have been developed to test and compare the untangling capabilities of the considered objective functions. In the experiments, a sample of highly distorted hexahedral elements is optimized with the proposed objective functions, and the rate of success of each function is obtained. The results of these experiments are presented and analyzed.
\end{abstract}

Keywords Hexahedral meshes · Mesh optimization · Mesh untangling

\section{Introduction}

For many simulation processes, hexahedral meshes present several numerical advantages over tetrahedral ones. For example, in finite element method simulations, hexahedral meshes are best suited to solve elastic, structural or fluid mechanics problems [1-3].

\section{J. I. López}

jilopez@siani.es

http://www.dca.iusiani.ulpgc.es/proyecto2015-2017/html/

index.html

1 University Institute for Intelligent Systems and Numerical Applications in Engineering, SIANI, University of Las Palmas de Gran Canaria, Las Palmas de Gran Canaria, Spain
The quality of the mesh has high repercussion on the numerical behavior of FEM. In some cases, mesh generators construct poor quality meshes or, even worse, with inverted elements. Indeed, a good quality mesh could degenerate into a mesh with inverted elements when the simulation requires nodes' movement. For this reason, it is of major importance to apply a mesh optimization algorithm to untangle the inverted elements and increase the quality of the mesh.

A widely used technique for mesh optimization consists in an iterative process in which each node is moved to a new position to improve the quality of the local mesh, which is the set of elements connected to the free node. This new position of the node is determined by minimizing certain objective function based on a distortion measure of the elements of the local mesh. In 2D, the optimization of a first-order quadrilateral element is carried out by decomposing the element in four triangles [4]. The non-degeneracy of these triangles is a necessary and sufficient condition for the validity of a bilinear element. However, for 3D case, necessary and sufficient conditions for the validity of a trilinear element are not known. Hence, it is not clear how to construct an objective function for hexahedral mesh optimization. In this work, we study several options for this issue. On the one hand, we form the objective function through the decomposition of the hexahedral elements into tetrahedra [5-7]. On the other hand, the objective function is built up from a shape distortion measure derived from the trilinear mapping between the reference and physical hexahedron [8].

Although the analyzed objective functions are able to optimize a large variety of meshes, some of them could not work properly for highly distorted elements. We have designed two computational experiments to compare the untangling capabilities of the proposed objective functions. 
Fig. 1 Master element $\hat{\Omega}$ and physical element $\Omega$

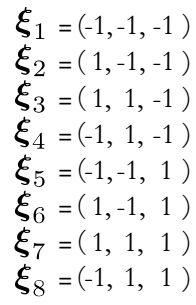

In the experiments, a sample of highly distorted hexahedral elements is optimized with the studied objective functions, and the rate of success of each function is obtained.

This paper is organized as follows. In Sect. 2, some preliminary concepts are given. The construction of objective functions through the decomposition of the element into tetrahedra is explained in Sect. 3. In Sect. 4, we propose an objective function constructed using a shape distortion measure derived from the trilinear mapping. In Sect. 5, the experiments are explained, and the results are discussed in detail. Finally, conclusions and future work are given in Sect. 6.

\section{Preliminary concepts}

In this paper, we will consider conformal meshes formed by first-order hexahedral elements defined by the trilinear transformation

$\mathbf{x}(\boldsymbol{\xi})=(x(\xi, \eta, \zeta), y(\xi, \eta, \zeta), z(\xi, \eta, \zeta))=\sum_{i=1}^{8} \mathbf{x}_{i} N_{i}(\xi)$

that maps the master element $\hat{\Omega}=[-1,1]^{3}$ with vertexes $\boldsymbol{\xi}_{i}$ into the physical hexahedron $\Omega$ with vertexes $\mathbf{x}_{i} \in \mathbb{R}^{3}$, see Fig. 1, where $N_{i}$ are the trilinear shape functions

$N_{i}(\xi)=\frac{1}{8}\left(1+\xi \xi_{i}\right)\left(1+\eta \eta_{i}\right)\left(1+\zeta \zeta_{i}\right)$,

that verify $N_{i}\left(\boldsymbol{\xi}_{j}\right)=\delta_{i j}$.

Here, we consider that a hexahedral element is valid if its Jacobian determinant is strictly positive, i.e., $J(\xi)>0$, $\xi \in[-1,1]^{3}$, where $J(\xi)=\operatorname{det}\left(\frac{\partial \mathbf{x}}{\partial \xi}\right)$. Thus, we assume that during the mesh generation process, the positivity of the Jacobian determinant of the trilinear mapping is pursued. We say that an element is invalid if its Jacobian takes negative values or equals zero at some point of the element. Sometimes, we refer to the invalid element also as tangled element or degenerate element.

It is known that, unlike 2D bilinear element, the positivity of the Jacobian at the corner points does not guarantee the validity of the trilinear element. Moreover, Jacobian positivity along the edges is not sufficient for its positivity in the whole element, see [9]. Figure 2 shows an example of

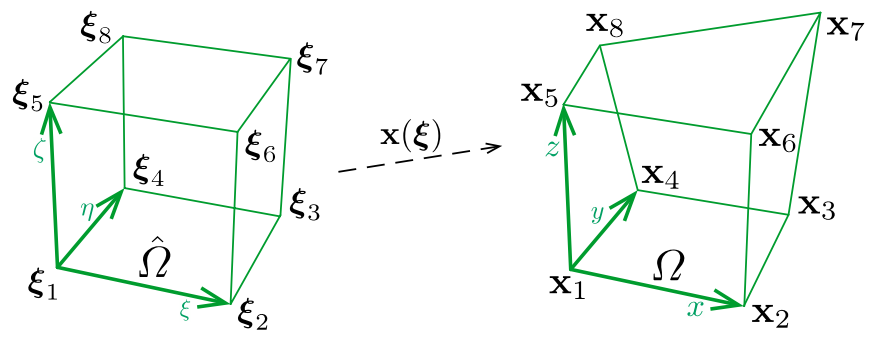

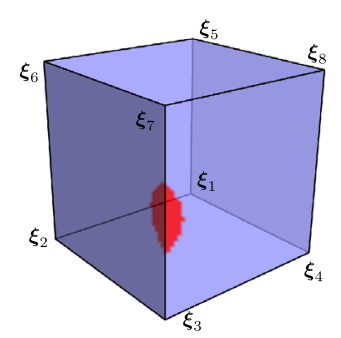

(a)

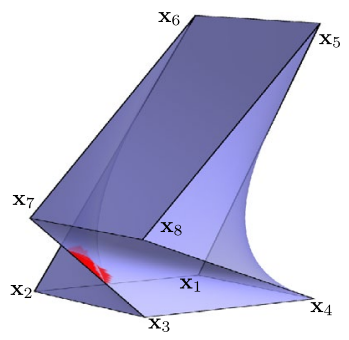

(b)
Fig. 2 Example of an invalid element with positive Jacobian in all corner points. a Master element, b physical element. Red area indicates the region of negative Jacobian

trilinear element with strictly positive Jacobian at all eight corners. However, its Jacobian takes negative values in some points of the element. According to the notation of Fig. 1, the coordinates of the vertexes of the physical element are $\mathbf{x}_{1}=(-1,-1,-1), \mathbf{x}_{2}=(1,-1,-1), \mathbf{x}_{3}=(1,1,-1)$, $\mathbf{x}_{4}=(-1,1,-1), \mathbf{x}_{5}=(-1,1,6), \mathbf{x}_{6}=(-1,-1,6)$, $\mathbf{x}_{7}=(1,-1,1)$ and $\mathbf{x}_{8}=(1,1,1)$.

Since necessary and sufficient conditions for the validity of a trilinear element are not known, it is not clear how to construct an objective function for hexahedral mesh optimization. There are some sufficient conditions for validity of a trilinear element that can be used for mesh optimization. Also, mesh optimization can be based on some necessary conditions for validity of a trilinear element. The objective of the present work is to analyze and compare the options for hexahedral mesh optimization.

\section{Optimization based on tetrahedral decomposition of a hexahedron}

Hexahedral mesh optimization can be converted into a tetrahedral optimization problem by decomposing each hexahedron in a set of tetrahedra. However, there is not a set of necessary and sufficient conditions based on tetrahedra decomposition that guarantees the validity of a hexahedron. In this section, we first introduce the quality and distortion measures for a tetrahedron. Then, different objective functions for hexahedral mesh optimization are defined by imposing sufficient or necessary conditions for the validity of a hexahedron. 


\subsection{Quality and distortion measures for tetrahedra}

Here, we summarize the formulation of the algebraic quality and distortion measures proposed by Knupp [4] for a tetrahedron. These measures are defined in terms of the deviation from an ideal tetrahedron that represents the desired shape to achieve.

Let $\tau$ be a tetrahedron whose vertexes are given by $\mathbf{x}_{k}=\left(x_{k}, y_{k}, z_{k}\right)^{T} \in \mathbb{R}^{3}, \quad k=0, \ldots, 3, \quad$ and $\tau_{R}$ be the reference tetrahedron with vertexes $\mathbf{u}_{0}=(0,0,0)^{T}$, $\mathbf{u}_{1}=(1,0,0)^{T}, \mathbf{u}_{2}=(0,1,0)^{T}$, and $\mathbf{u}_{3}=(0,0,1)^{T}$. If we choose $\mathbf{x}_{0}$ as the translation vector, the affine map that takes $\tau_{R}$ to $\tau$ is $\mathbf{x}=A \mathbf{u}+\mathbf{x}_{0}$, where $A$ is the Jacobian matrix of the affine map referenced to node $\mathbf{x}_{0}$, and expressed as $A=\left(\mathbf{x}_{1}-\mathbf{x}_{0}, \mathbf{x}_{2}-\mathbf{x}_{0}, \mathbf{x}_{3}-\mathbf{x}_{0}\right)$.

Let us consider that $\tau_{I}$ is our ideal or target tetrahedron whose vertexes are $\mathbf{v}_{0}, \mathbf{v}_{1}, \mathbf{v}_{2}$, and $\mathbf{v}_{3}$. If we take $\mathbf{v}_{0}=(0,0,0)^{T}$, the linear map that takes $\tau_{R}$ to $\tau_{I}$ is $\mathbf{v}=W \mathbf{u}$, where $W=\left(\mathbf{v}_{1}, \mathbf{v}_{2}, \mathbf{v}_{3}\right)$ is its Jacobian matrix.

Affine map that takes $\tau_{I}$ to $\tau$ is given by $\mathbf{x}=A W^{-1} \mathbf{v}+\mathbf{x}_{0}$, and its Jacobian matrix is $S=A W^{-1}$. Quality metrics of the tetrahedron $\tau$ can be defined in terms of the matrix $S$. For example, the mean ratio

$q=\frac{3 \sigma^{2 / 3}}{\|S\|^{2}}$

is an easily computable algebraic quality metric of $\tau$, where $\sigma=\operatorname{det}(S)$ and $\|S\|$ is the Frobenius norm of $S$. The maximum value of $q$ is the unity, and it is reached when $A=\mu R W$, where $\mu$ is a scalar, and $R$ is a rotation matrix. In other words, $q$ is maximum if and only if $\tau$ and $\tau_{I}$ are similar tetrahedra. Besides, any flat tetrahedron has quality measure zero.

The distortion measure for a tetrahedron is defined as the inverse of its quality, i.e., $\eta=1 / q$. The distortion $\eta$ is equal to 1 for the ideal tetrahedron and tends to $\infty$ when the tetrahedron tends to be degenerated. The objective function to be minimized is defined as $K=\sum_{i=1}^{N} \eta_{i}^{p}$, where $N$ is the number of tetrahedra in the local mesh and, usually, $p=1$ or $p=2$.

The objective function $K$ becomes discontinuous when the volume of any tetrahedron tends to zero. Due to these singularities, the function $K$ improves the quality of valid elements, but it does not work properly when the mesh is tangled $(\sigma \leq 0)$. In [10], we proposed a modification of $K$ by replacing $\sigma$ by the positive and increasing function $h(\sigma)=\frac{1}{2}\left(\sigma+\sqrt{\sigma^{2}+4 \delta^{2}}\right)$. Then, the modified distortion becomes

$\eta^{*}=\frac{\|S\|^{2}}{3 h(\sigma)^{2 / 3}}$
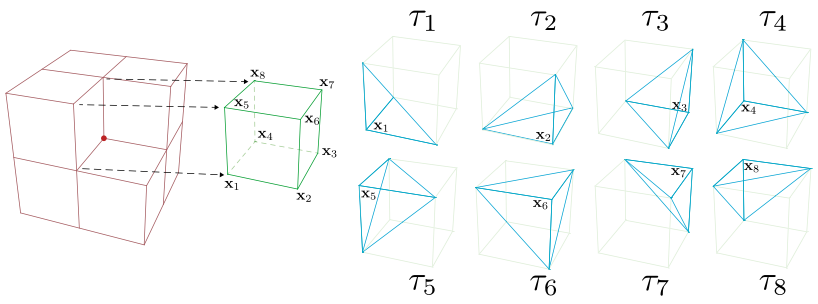

Fig. 3 On the left, an example of a hexahedral local mesh composed by eight elements $(M=8)$ where the red point is the free node. On the right, the tetrahedra decomposition proposed by Knupp for a hexahedron

This modification eliminates the barriers associated with their singularities, and the new objective function $K^{*}=\sum_{i=1}^{N}\left(\eta_{i}^{*}\right)^{p}$ becomes smooth in $\mathbb{R}^{3}$. In the feasible region (subset of $\mathbb{R}^{3}$ where the free node could be placed for the local mesh to be valid), the modified objective function $K^{*}$ approximates the original function $K$ as $\delta \rightarrow 0$ and then, the minimum of the original and modified objective functions are nearly identical when $\delta$ is small. When this region does not exist, the minimum of the modified objective function is located in such a way that it tends to untangle the local mesh. Thus, the modified objective function allows the simultaneous untangling and smoothing of the mesh. The value of $\delta$ is selected in terms of the local mesh under consideration, making it as small as possible and in such a way that the evaluation of the minimum of the modified function does not present any computational problem. For more details, see $[10,11]$. The unconstrained optimization problem can be easily solved with any standard method, see for example [12].

\subsection{Objective function for a hexahedral mesh based on necessary conditions}

Knupp proposes a distortion measure for a hexahedral element based on its decomposition in eight tetrahedra $[5,13]$. The tetrahedron $\tau_{i}$ is the one formed by the $i$-th vertex of the hexahedron and its edges coincident on this vertex, see Fig. 3. Let us consider the Jacobian matrix $A_{i}$, the weighted Jacobian matrix $S_{i}=A_{i} W_{i}^{-1}$, and the distortion $\eta\left(S_{i}\right)$ of the tetrahedron $\tau_{i}$. Then, the distortion of the hexahedron is given by

$\eta=\frac{1}{8} \sum_{i=1}^{8} \eta\left(S_{i}\right)=\frac{1}{8} \sum_{i=1}^{8} \frac{\left\|S_{i}\right\|^{2}}{3 \sigma\left(S_{i}\right)^{2 / 3}}$.

In our case, the ideal hexahedron is the cube, and therefore, the ideal tetrahedron is the rectangular isosceles. Note that $1 \leq \eta<\infty$ being $\eta=1$ for a cube.

The modified distortion of a hexahedron is defined by 


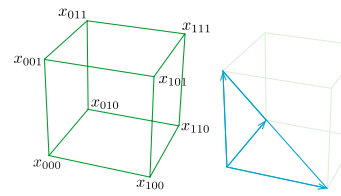

(a)

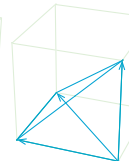

(c)

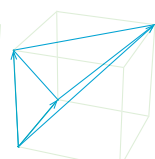

(d)

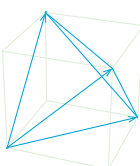

(e)
Fig. 4 Examples of tetrahedra for sufficient conditions. a Vertex enumeration, $\mathbf{b} \alpha_{000}, \mathbf{c} \beta_{000}^{1}, \mathbf{d} \gamma_{000}^{1}$, and $\mathbf{e} \kappa_{000}$

$\eta^{*}=\frac{1}{8} \sum_{i=1}^{8} \eta^{*}\left(S_{i}\right)=\frac{1}{8} \sum_{i=1}^{8} \frac{\left\|S_{i}\right\|^{2}}{3 h\left(\sigma\left(S_{i}\right)\right)^{2 / 3}}$.

Let us consider the free node $\mathbf{x}$ of a hexahedral local mesh. Then, following [6], the modified objective function for this local mesh is

$K^{*}(\mathbf{x})=\frac{1}{M} \sum_{m=1}^{M}\left(\eta_{m}^{*}\right)^{p}(\mathbf{x})$,

where $M$ is the number of hexahedra connected to the free node $\mathbf{x}$, and $\eta_{m}^{*}$ is the modified distortion of the $m$-th hexahedron, see Fig. 3.

As we previously said, a hexahedron is valid if $J(\xi)>0$, $\xi \in[-1,1]^{3}$ and, in particular, this must be true for all the vertexes $\xi_{i}$ of the hexahedron. It is easy to prove that the Jacobian matrix of the trilinear mapping $\mathbf{x}(\boldsymbol{\xi})$ evaluated at the vertexes $\xi_{i}$ is related to the tetrahedra matrix $S_{i}$ as follows: $\left.\left(\frac{\partial \mathbf{x}}{\partial \xi}\right)\right|_{\xi=\xi_{i}}=\mu S_{i}$, where $\mu$ is a positive scalar. Therefore, $\operatorname{det}\left(S_{i}\right)>0$ are necessary conditions for the validity of the hexahedron.

\subsection{Objective function for a hexahedral mesh based on sufficient conditions}

Ushakova [7] proposes a set of sufficient conditions that guarantee the non-degeneracy of a hexahedron. These conditions are formulated in terms of the volumes of certain tetrahedra constructed from the vertexes $\mathbf{x}_{i_{1} i_{2} i_{3}}$ of the hexahedron, see Fig. 4 . The tetrahedra are grouped in four groups $\alpha, \beta, \gamma$, and $\kappa$ :

$$
\begin{aligned}
& \alpha_{i_{1} i_{2} i_{3}}=\left(\mathbf{x}_{i_{1} i_{2} i_{3}}, \mathbf{x}_{\bar{i}_{1} i_{2} i_{3}}, \mathbf{x}_{i_{1} \bar{i}_{2} i_{3}}, \mathbf{x}_{i_{1} i_{2} \bar{i}_{3}}\right), \\
& \beta_{i_{1} i_{2} i_{3}}^{1}=\beta_{i_{2} i_{3}}^{1 i_{1}}=\left(\mathbf{x}_{i_{1} i_{2} i_{3}}, \mathbf{x}_{i_{i_{1}} i_{2} i_{3}}, \mathbf{x}_{i_{1} \bar{i}_{2} i_{3}}, \mathbf{x}_{i_{1} i_{2} \bar{i}_{3}}\right), \\
& \beta_{i_{1} i_{2} i_{3}}^{2}=\beta_{i_{3} i_{1}}^{2 i_{2}}=\left(\mathbf{x}_{i_{1} i_{2} i_{3}}, \mathbf{x}_{i_{1} i_{1} i_{3}}, \mathbf{x}_{i_{1} \bar{i}_{2} i_{3}}, \mathbf{x}_{i_{1} \bar{i}_{2} \bar{i}_{3}}\right), \\
& \beta_{i_{1} i_{2} i_{3}}^{3}=\beta_{i_{1} i_{2}}^{3 i_{3}}=\left(\mathbf{x}_{i_{1} i_{2} i_{3}}, \mathbf{x}_{i_{1} i_{2} i_{3}}^{-}, \mathbf{x}_{i_{1} \bar{i}_{2} \bar{i}_{3}}, \mathbf{x}_{i_{1} i_{2} \bar{i}_{3}}\right), \\
& \gamma_{i_{1} i_{2} i_{3}}^{1}=\gamma_{i_{2} i_{3}}^{1 i_{1}}=\left(\mathbf{x}_{i_{1} i_{2} i_{3}}, \mathbf{x}_{i_{1} \bar{i}_{2} \bar{i}_{3}}, \mathbf{x}_{i_{1} \bar{i}_{2} i_{3}}, \mathbf{x}_{i_{1} i_{2} \bar{i}_{3}}\right), \\
& \gamma_{i_{1} i_{2} i_{3}}^{2}=\gamma_{i_{3} i_{1}}^{2 i_{1}}=\left(\mathbf{x}_{i_{1} i_{2} i_{3}}, \mathbf{x}_{\bar{i}_{1} i_{2} i_{3}}, \mathbf{x}_{i_{1} \bar{i}_{2} \bar{i}_{3}}, \mathbf{x}_{i_{1} i_{2} \bar{i}_{3}}\right), \\
& \gamma_{i_{1} i_{2} i_{3}}^{3}=\gamma_{i_{1} i_{2}}^{3 i_{3}}=\left(\mathbf{x}_{i_{1} i_{2} i_{3}}, \mathbf{x}_{\bar{i}_{1} i_{2} i_{3}}, \mathbf{x}_{i_{1} \bar{i}_{2} i_{3}}, \mathbf{x}_{\bar{i}_{1} \bar{i}_{2} \bar{i}_{3}}\right),
\end{aligned}
$$

$\kappa_{i_{1}}=\left(\mathbf{x}_{i_{1} i_{1} i_{1}}, \mathbf{x}_{i_{1} \bar{i}_{1} \bar{i}_{1}}, \mathbf{x}_{\bar{i}_{1} i_{1} \bar{i}_{1}}, \mathbf{x}_{\bar{i}_{1} \bar{i}_{1} i_{1}}\right)$,

where $i_{1}, i_{2}, i_{3}=0,1$ and $\overline{0}=1, \overline{1}=0$.

Tetrahedra $\alpha$ are formed by three edges with the common corner (eight tetrahedra), tetrahedra $\beta$ by two edges and the diagonal of one of adjacent faces (24 tetrahedra), tetrahedra $\gamma$ by two edges and the inner diagonal of the hexahedron (24 tetrahedra), and tetrahedra $\kappa$ by diagonals of faces (two tetrahedra). See some samples in Fig. 4. The total number of tetrahedra is $8+24+24+2=58$. Note that the eight tetrahedra $\alpha$ are the same eight tetrahedra proposed by Knupp for optimization of a hexahedron.

Non-degeneracy sufficient conditions are expressed in form of 27 inequalities [7]:

$\operatorname{det}\left(A\left(\alpha_{i_{1} i_{2} i_{3}}\right)\right) \geq 0, \quad i_{1}, i_{2}, i_{3}=0,1 ;$

$\sum_{i_{k}=0}^{1} \operatorname{det}\left(A\left(\beta_{i_{l} i_{m}}^{k i_{k}}\right)\right) \geq 0, \quad(k l m)=(123), i_{l}, i_{m}=0,1 ;$

$\sum_{i_{l}, i_{m}=0}^{1} \operatorname{det}\left(A\left(\gamma_{i_{l} i_{m}}^{k i_{k}}\right)\right) \geq 0, \quad(k l m)=(123), i_{k}=0,1 ;$

$\sum_{i_{1}=0}^{1} \operatorname{det}\left(A\left(\kappa_{i_{1}}\right)\right) \geq 0$

where $A(\tau)$ is the Jacobian matrix of the tetrahedron $\tau$, taking as reference node the first one in the list of vertexes that defines $\tau$.

Among 27 inequalities, we have eight inequalities (5) for non-degeneracy in the vertexes, 12 inequalities (6) for non-degeneracy on edges, six inequalities (7) for nondegeneracy on faces, and one inequality (8) for non-degeneracy in the inner part of a hexahedron.

As pointed in [7], these non-degeneracy conditions are difficult to impose in a mesh generation process, because they involve sum of volumes. For this reason, Ushakova proposes other sets of conditions, easier to impose, based on the non-degeneracy of some of the previously related tetrahedra. For example, one of the proposed tests checks the validity of $\alpha$ and $\beta$ tetrahedra

$$
\begin{array}{r}
\operatorname{det}\left(A\left(\alpha_{i_{1} i_{2} i_{3}}\right)\right)>0, \quad \operatorname{det}\left(A\left(\beta_{i_{1} i_{2} i_{3}}^{k}\right)\right)>0, \\
i_{1}, i_{2}, i_{3}=0,1, k=1,2,3 .
\end{array}
$$

Note that only the non-degeneracy of the tetrahedra $\alpha$ are necessary conditions for the validity of the hexahedron. The non-degeneracy of the remainder individual tetrahedra $\beta, \gamma$, and $\kappa$, are not necessary conditions. 
Fig. 5 Barrier functions. a $h(\sigma)^{-1}$ with different values of $\delta$. $\mathbf{b} h^{\prime}(\sigma)^{-1}$ with different values of $\delta$. $\mathbf{c}$ Comparison of $h(\sigma)^{-1}$ and $h^{\prime}(\sigma)^{-1}$ with $\delta=0.25$

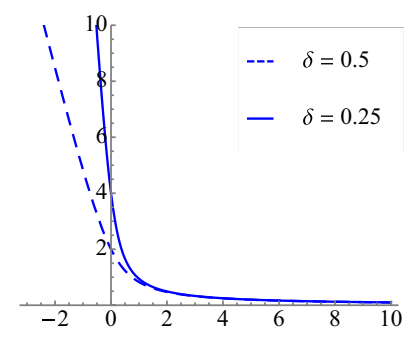

(a)

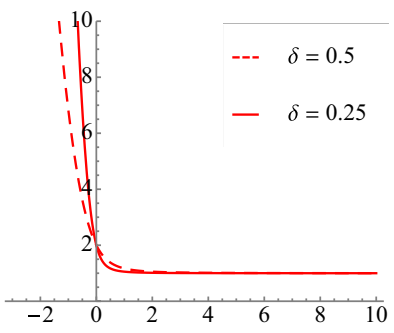

(b)

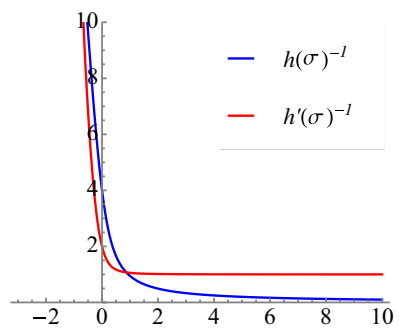

(c)

\subsubsection{Proposed objective functions}

Here, we present objective functions based on the two types of conditions given by (5)-(8) and (9).

In (5)-(8), we differentiate two types of conditions. Conditions (5) involve individual tetrahedra, and conditions (6)-(8) concern several tetrahedra simultaneously. Equation (5) is the set of necessary conditions mentioned in Sect. 3.2, so, for them, we propose the same terms as in (2), see first summation in Eq. (10). For conditions (6)-(8), we have added additional terms to guarantee the validity of the hexahedron, see second, third, and fourth summations in Eq. (10). These conditions could be imposed by means of the function $h(\sigma)$. For example, the term associated to the condition $\operatorname{det}\left(A\left(\beta_{00}^{10}\right)\right)+\operatorname{det}\left(A\left(\beta_{00}^{11}\right)\right)>0$ could be $h\left(\operatorname{det}\left(A\left(\beta_{00}^{10}\right)\right)+\operatorname{det}\left(A\left(\beta_{00}^{11}\right)\right)\right)^{-1}$. Nevertheless, function $h(\sigma)^{-1}$ is not dimensionless, and then, it is sensitive to scale changes. For this reason, we propose $h^{\prime}(\sigma)^{-1}=2-\frac{\sigma}{h(\sigma)}$ to impose conditions (6)-(8). This function has a similar behavior to $h(\sigma)^{-1}$, see Fig. 5, but it is dimensionless and scale invariant when $\delta \rightarrow 0$.

Let us consider $S(\tau)$ the weighted Jacobian matrix of a tetrahedron $\tau$, taking as reference tetrahedron its counterpart in the master hexahedron, and $\sigma(\tau)=\operatorname{det}(S(\tau))$. Then, the proposed objective function to impose conditions (5)(8) for a hexahedron is

$$
\begin{aligned}
k^{*}= & \sum_{i_{1}, i_{2}, i_{3}} \frac{\left\|S\left(\alpha_{i_{1} i_{2} i_{3}}\right)\right\|^{2}}{3 h\left(\sigma\left(\alpha_{i_{1} i_{2} i_{3}}\right)\right)^{2 / 3}}+\sum_{k, i_{l}, i_{m}} \frac{1}{h^{\prime}\left(\sum_{i_{k}} \sigma\left(\beta_{i_{l} i_{m}}^{k i_{k}}\right)\right)} \\
& +\sum_{k, i_{k}} \frac{1}{h^{\prime}\left(\sum_{i_{l}, i_{m}} \sigma\left(\gamma_{i_{l} i_{m}}^{k i_{k}}\right)\right)}+\frac{1}{h^{\prime}\left(\sum_{i_{1}} \sigma\left(\kappa_{i_{1}}\right)\right)},
\end{aligned}
$$

where $i_{1}, i_{2}, i_{3}=0,1,(\mathrm{klm})=(123)$ and $i_{l}, i_{m}, i_{k}=0,1$. Note that the first term of (10) tends to improve the quality of the tetrahedra $\alpha$, and the rest of the terms just impose barriers associated to conditions (6)-(8).

For conditions like (9), which only involve individual tetrahedra, the objective function for a hexahedron is

$$
k^{*}=\sum_{i_{1}, i_{2}, i_{3}} \frac{\left\|S\left(\alpha_{i_{1} i_{2} i_{3}}\right)\right\|^{2}}{3 h\left(\sigma\left(\alpha_{i_{1} i_{2} i_{3}}\right)\right)^{2 / 3}}+\sum_{k, i_{1}, i_{2}, i_{3}} \frac{\left\|S\left(\beta_{i_{1} i_{2} i_{3}}^{k}\right)\right\|^{2}}{3 h\left(\sigma\left(\beta_{i_{1} i_{2} i_{3}}^{k}\right)\right)^{2 / 3}}
$$

where $i_{1}, i_{2}, i_{3}=0,1$ and $k=1,2,3$.

Finally, either for conditions (5)-(8) or (9), the objective function for a local hexahedral mesh is

$K^{*}(\mathbf{x})=\frac{1}{M} \sum_{m=1}^{M}\left(k_{m}^{*}\right)^{p}(\mathbf{x})$

where $M$ is the number of hexahedra connected to the free node $\mathbf{x}$, and $k_{m}^{*}$ is the term associated to the $m$-th hexahedron of the local mesh.

\section{Optimization based on the global distortion of the hexahedron}

An objective function for a hexahedral mesh can be defined through a global distortion measure for a hexahedron, as proposed in [8].

We can define the modified pointwise distortion as in (2), but now, considering that $S(\boldsymbol{\xi})=(\partial \mathbf{x} / \partial \boldsymbol{\xi}) W(\boldsymbol{\xi})^{-1}$ is the weighted Jacobian matrix of the trilinear transformation (1), where $\partial \mathbf{x} / \partial \boldsymbol{\xi}$ is the Jacobian matrix of the mapping from the master element to the physical one, and $W(\xi)$ is Jacobian matrix of the mapping from master element to the ideal (target) one. In our case, the ideal hexahedron coincides with the master element $\hat{\Omega}=[-1,1]^{3}$, so the matrix $W(\xi)$ is the unit matrix, and thus, $S(\boldsymbol{\xi})=(\partial \mathbf{x} / \partial \xi)$.

Then, the pointwise modified distortion for a hexahedron is defined as

$\eta^{*}(\xi)=\frac{\|S(\xi)\|^{2}}{3 h(\sigma(\xi))^{2 / 3}}, \quad \xi \in[-1,1]^{3}$

where $\sigma(\xi)=\operatorname{det}(S(\xi))$.

The pointwise distortion $\eta^{*}(\xi)$ attains values between 1 and $\infty$. If the physical element $\Omega$ is a cube, then $S(\xi)=\mu I$, and in this case, $\eta^{*}(\xi) \rightarrow 1$ when $\delta \rightarrow 0$. On the other hand, if $\sigma(\xi) \leq 0$ for some $\xi$, then $\eta^{*}(\xi) \rightarrow \infty$ when $\delta \rightarrow 0$.

The global modified distortion measure of a hexahedron $\Omega$ is given by

$\eta_{\Omega}^{*}=\frac{1}{V_{\hat{\Omega}}} \int_{\hat{\Omega}} \eta^{*}(\xi) d \hat{\Omega}$, 
where $V_{\hat{\Omega}}=8$ is the volume of $\hat{\Omega}$. In [8], the quality of a hexahedron is defined as $\left(\eta_{\Omega}^{*}\right)^{-1}$ with $\delta \rightarrow 0$.

This distortion measure presents pseudo-barriers, that is, $\eta_{\Omega}^{*} \rightarrow \infty$ when $\sigma(\xi) \leq 0$ and $\delta \rightarrow 0$. Then, we define the objective function for a local mesh as

$K^{*}(\mathbf{x})=\frac{1}{M} \sum_{m=1}^{M} \eta_{\Omega_{m}}^{*}(\mathbf{x})$,

where $M$ is the number of hexahedra connected to the free node $\mathbf{x}$.

The integral is evaluated using a numerical quadrature rule. It is important to highlight that the objective function (14) does not work properly when the quadrature rule "does not see" the pseudo-singularities of $\eta_{\Omega}^{*}$, that is, there is not any quadrature point inside the region where $\eta_{\Omega}^{*}$ takes very high values.

\subsection{Numerical quadrature of the distortion}

We use the simple quadrature rule given by

$\int_{\hat{\Omega}} \eta^{*}(\boldsymbol{\xi}) d \hat{\Omega} \approx \sum_{i, j, k=1}^{2} w_{i j k} \eta^{*}\left(\boldsymbol{\xi}_{i j k}\right)$,

where $\xi_{i j k}$ are the vertexes of the master element $\hat{\Omega}$ and $w_{i j k}=\frac{1}{8} \operatorname{Vol}(\hat{\Omega})$. It is reasonable to place quadrature points on the vertexes, because the singularities are usually close to the boundary of the $\hat{\Omega}$.

In some cases, the optimization of a highly distorted hexahedron by means of the function (14) may not untangle the element. This is because the numerical evaluation of the distortion is not accurate enough. To improve the precision of the numerical integration, we increase the number of quadrature points subdividing the element and performing the integration on each sub-element using the same quadrature rule given by (15). This subdivision can be global or local. Global subdivisions can be computationally impractical, because the number of terms in the objective function increases exponentially when several subdivisions steps are required. We propose an adaptive quadrature scheme using an octree subdivision.

\subsection{Adaptive numerical quadrature of the hexahedron distortion}

The strategy for adaptive quadrature is based on dividing only sub-elements of $\hat{\Omega}$ where the Jacobian is negative. In this way, quadrature points tend to be concentrated in the neighborhood of the pseudo-singularities of $\eta^{*}(\xi)$.

The algorithm (1) describes the optimization procedure using adaptive quadrature.

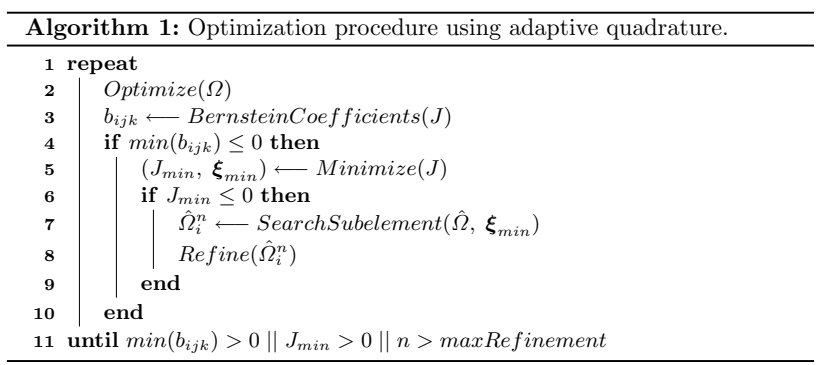

The procedure consists in a repeat loop. In a first step (line 2), the hexahedron $\Omega$ is optimized. The first time that $\Omega$ is optimized, we take as quadrature points the vertexes of $\hat{\Omega}$. In the second step of the algorithm (line 3), the control values $b_{i j k}$ of the expansion of the Jacobian $J$ in Bernstein polynomials are computed as explained in Appendix A. If the lower bound of $J, \min \left(b_{i j k}\right)$, is not positive (line 4), then the validity of the element cannot be determined. In this case, the Jacobian is minimized (line 5), obtaining as result its minimum value $J_{\text {min }}$ and $\xi_{\text {min }}=\arg \min J(\xi), \xi \in \hat{\Omega}$. If $J_{\min } \leq 0$ (line 6), then the sub-element $\hat{\Omega}_{i}^{n}$ that contains $\boldsymbol{\xi}_{\min }$ (line 7) is refined (line 8). The superscript $n$ indicates the level of refinement of this sub-element $\left(\hat{\Omega}=\hat{\Omega}^{0}\right)$.

Note that there are three exit criteria for the loop (line 11). The first two criteria check if the element is already valid, either because the Jacobian is positively bounded $\left(\min \left(b_{i j k}\right)>0\right)$ or its minimum is positive $\left(J_{\min }>0\right)$. The third exit criterion ( $n>$ maxRefinement) avoids an infinity loop by limiting the maximum number of recursive refinements that can be made. In practice, we have observed that in most cases, five steps of refinement are enough. An example of two steps of adaptive quadrature in $2 \mathrm{D}$ is illustrated in Fig. 6.

When we deal with a local mesh instead of a single element, we apply the same adaptive quadrature to each element of the local mesh.

Remark 1 Note that with any quadrature rule, the minimization of the objective function (14) imposes necessary conditions for the validity of a hexahedron insomuch as it imposes the positivity of the Jacobian at the quadrature points. If the integration is performed without any subdivision, i.e., using the eight vertexes of the hexahedron as quadrature points, the objective function (14 is equivalent to the objective function (4), see the last paragraph of the Sect. 3.2. So, the objective function (4) is a particular case of (14). If the integration is performed with one subdivision, the positivity of the Jacobian is imposed in 27 quadrature points: 8 corners, center of each face, center of each edge, and the center of the element. 
Fig. 6 Example of two steps of adaptive quadrature in a $2 \mathrm{D}$ element. The red shaded $\hat{\Omega}_{i}^{n}$ is the sub-element that needs to be refined, because it contains $\boldsymbol{\xi}_{\text {min }}$. The black points are the quadrature points in each $\hat{\Omega}_{i}^{n}$
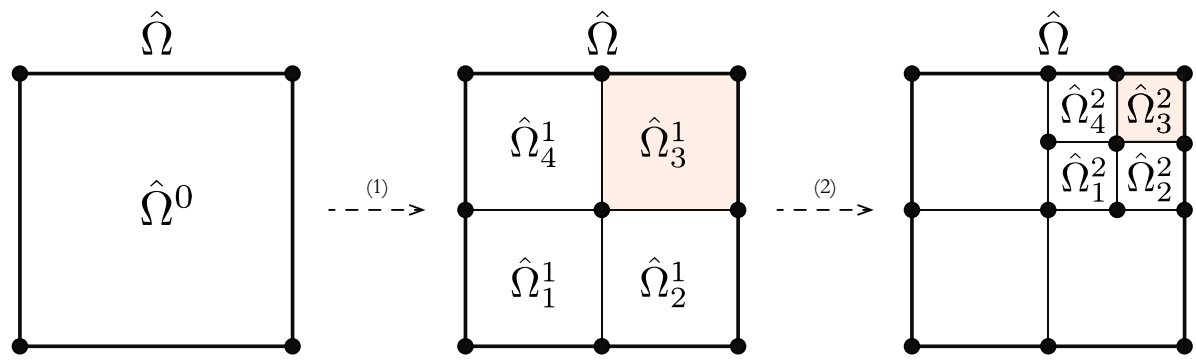

\section{Experiments and results}

The goal of the proposed experiments is to compare the untangling capability of different objective functions. We have considered three groups of objective functions: tetrahedron distortion (TD), sufficient conditions (SC), and global distortion (GD). The TD group consists of several objective functions based on the distortion of certain tetrahedra, SC is based on non-degeneracy sufficient conditions defined through tetrahedra volumes, and GD is based on a global distortion measure of a hexahedron defined through the trilinear transformation.

We compare the nine objective functions listed below:

1. $\mathrm{TD}_{\alpha}$ : Objective function based on the distortion of the tetrahedra $\alpha$.

2. $\mathrm{TD}_{\alpha \beta}$ : Objective function based on the distortion of the tetrahedra $\alpha$ and $\beta$.

3. $\mathrm{TD}_{\alpha \gamma}$ : Objective function based on the distortion of the tetrahedra $\alpha$ and $\gamma$.

4. $\mathrm{TD}_{\alpha \kappa}$ : Objective function based on the distortion of the tetrahedra $\alpha$ and $\kappa$.

5. $\mathrm{TD}_{\alpha \beta \gamma \kappa}$ : Objective function based on the distortion of the tetrahedra $\alpha, \beta, \gamma$ and $\kappa$.

6. SC: Objective function based on sufficient conditions.

7. $\mathrm{GD}_{0}$ : Objective function based on global distortion of the hexahedron, evaluated without any subdivision of $\hat{\Omega}$ (eight quadrature points).

8. $\mathrm{GD}_{1}$ : Objective function based on global distortion of the hexahedron, evaluated with one subdivision of $\hat{\Omega}$ (27 quadrature points).

9. $\mathrm{GD}_{n}$ : Objective function based on global distortion of the hexahedron, evaluated with adaptive quadrature for $\hat{\Omega}$.

The $\mathrm{TD}_{\alpha}$ objective function is given by Eq. (4), and the rest of $\mathrm{TD}^{\prime} s$ are based on Eqs. (11) and (12) adding the terms associated to the $\beta, \gamma$ or $\kappa$ tetrahedra. The SC is based on Eqs. (10) and (12). It is worth mentioning that $\mathrm{TD}_{\alpha \beta \gamma \kappa}$ also imposes sufficient conditions and that they are more restrictive than the conditions imposed by SC. Finally, the $\mathrm{GD}^{\prime} s$ are based on Eqs. (13), (14), and (15). Note that functions $\mathrm{TD}_{\alpha}$ and $\mathrm{GD}_{0}$ are equivalent, see Remark 1.

We have designed two computational experiments that are explained in the following sections.

\subsection{Experiment 1}

The first experiment consists in optimizing two specific isolated elements taken from [14], one invalid and another valid. Each element is optimized with the proposed objective functions considering $\mathbf{x}_{i}(i=1, \ldots, 8)$ as free node and fixing the remaining vertexes. Then, we check the validity of the optimized elements.

Table 1 shows the results of this experiment. Element 1 is formed by the vertexes $\mathbf{x}_{1}=(0,0,0), \mathbf{x}_{2}=$ $(1,0,0), \mathbf{x}_{3}=(1.5,1.25,0), \mathbf{x}_{4}=(0,1,0), \mathbf{x}_{5}=(0,0,1)$, $\mathbf{x}_{6}=(1,-0.5,1), \mathbf{x}_{7}=(1,0.5,0.5)$ and $\mathbf{x}_{8}=(-0.5,1,1)$, see Fig. 7 a.

This element is initially invalid, so it is not possible to guarantee that it has a feasible region when certain vertexes act as a free node. This is due to the fact that the degeneration of the hexahedron can be produced by the position of the other vertexes. For example, this occurs when the vertexes $\mathbf{x}_{1}, \mathbf{x}_{2}, \mathbf{x}_{4}$ or $\mathbf{x}_{5}$ are taken as free nodes. If the free node is another vertex, the validity of the optimized hexahedron depends on the chosen objective function. For example, we can see that $\mathrm{TD}_{\alpha \gamma}$ does not obtain a valid element when the free node is the vertex $\mathbf{x}_{8}$, but using $\mathrm{GD}_{n}$, the optimized element is valid, see Fig. 7b, c.

The vertexes of the element 2 are $\mathbf{x}_{1}=(0,0,0), \mathbf{x}_{2}=$ $(1,0,0), \mathbf{x}_{3}=(1.5,0.5,0), \mathbf{x}_{4}=(0,1,0), \mathbf{x}_{5}=(0,0,1)$, $\mathbf{x}_{6}=(0.5,0.5,1.25), \mathbf{x}_{7}=(1,0.75,0.5)$ and $\mathbf{x}_{8}=(0.25$, $(0.5,1.5)$, see Fig. $7 \mathrm{~d}$. This is a valid element, so it always exists a feasible region regardless of the vertex taken as free node. Nevertheless, the optimization with some objective functions produces an invalid element, as for example $\mathrm{TD}_{\alpha \gamma}$ with the vertex $\mathbf{x}_{4}$ as free node, see Fig. 7e. Instead, using, for example, $\mathrm{GD}_{n}$, the resulting element is always valid, see Fig. 7f. 
Fig. 7 Elements for the experiment 1 . a Initial invalid element 1. b Resulting invalid element 1 after relocating the node $\mathbf{x}_{8}$ using $\mathrm{TD}_{\alpha \gamma}$. $\mathbf{c}$ Resulting valid element 1 after relocating $\mathbf{x}_{8}$ using $\mathrm{GD}_{n}$. $\mathbf{d}$ Initial valid element 2. e Resulting invalid element 2 after relocating $\mathbf{x}_{4}$ using $\mathrm{TD}_{\alpha \gamma}$. e Resulting valid element 2 after relocating $\mathbf{x}_{4}$ using $\mathrm{GD}_{n}$

Table 1 Experiment 1 results

\begin{tabular}{|c|c|c|c|c|c|c|c|c|}
\hline \multirow[t]{2}{*}{ Obj. function } & \multicolumn{8}{|c|}{ Free node } \\
\hline & $\mathbf{x}_{1}$ & $\mathbf{x}_{2}$ & $\mathbf{x}_{3}$ & $\mathbf{x}_{4}$ & $\mathbf{x}_{5}$ & $\mathbf{x}_{6}$ & $\mathbf{x}_{7}$ & $\mathbf{x}_{8}$ \\
\hline & \multicolumn{8}{|c|}{ Element 1} \\
\hline $\mathrm{TD}_{\alpha}\left(\mathrm{GD}_{0}\right)$ & $\times$ & $\times$ & $\checkmark$ & $x$ & $\times$ & $\checkmark$ & $\checkmark$ & $\checkmark$ \\
\hline $\mathrm{TD}_{\alpha \beta}$ & $\times$ & $\times$ & $\checkmark$ & $\times$ & $\times$ & $\checkmark$ & $\checkmark$ & $\checkmark$ \\
\hline $\mathrm{TD}_{\alpha \gamma}$ & $\times$ & $x$ & $\checkmark$ & $\times$ & $\times$ & $\checkmark$ & $\checkmark$ & $x$ \\
\hline $\mathrm{TD}_{\alpha \kappa}$ & $x$ & $x$ & $\checkmark$ & $x$ & $x$ & $\checkmark$ & $\checkmark$ & $\checkmark$ \\
\hline $\mathrm{TD}_{\alpha \beta \gamma \kappa}$ & $\times$ & $x$ & $\checkmark$ & $x$ & $x$ & $\checkmark$ & $\checkmark$ & $\times$ \\
\hline$S C$ & $\times$ & $x$ & $\checkmark$ & $\times$ & $\times$ & $\checkmark$ & $\checkmark$ & $\checkmark$ \\
\hline $\mathrm{GD}_{1}$ & $\times$ & $x$ & $\checkmark$ & $x$ & $x$ & $\checkmark$ & $\checkmark$ & $\checkmark$ \\
\hline \multirow[t]{2}{*}{$\mathrm{GD}_{n}$} & $x$ & $x$ & $\checkmark$ & $x$ & $x$ & $\checkmark$ & $\checkmark$ & $\checkmark$ \\
\hline & \multicolumn{8}{|c|}{ Element 2} \\
\hline $\mathrm{TD}_{\alpha}\left(\mathrm{GD}_{0}\right)$ & $\checkmark$ & $\checkmark$ & $\checkmark$ & $x$ & $x$ & $\checkmark$ & $\times$ & $\checkmark$ \\
\hline $\mathrm{TD}_{\alpha \beta}$ & $\checkmark$ & $\checkmark$ & $\checkmark$ & $\checkmark$ & $\checkmark$ & $\checkmark$ & $\checkmark$ & $\checkmark$ \\
\hline $\mathrm{TD}_{\alpha \gamma}$ & $\checkmark$ & $\times$ & $\checkmark$ & $\times$ & $\checkmark$ & $\checkmark$ & $\checkmark$ & $\checkmark$ \\
\hline $\mathrm{TD}_{\alpha \kappa}$ & $\checkmark$ & $\checkmark$ & $\checkmark$ & $x$ & $x$ & $\checkmark$ & $\times$ & $\checkmark$ \\
\hline $\mathrm{TD}_{\alpha \beta \gamma \kappa}$ & $\checkmark$ & $\checkmark$ & $\checkmark$ & $x$ & $\checkmark$ & $\checkmark$ & $\checkmark$ & $\checkmark$ \\
\hline$S C$ & $\checkmark$ & $\checkmark$ & $\checkmark$ & $x$ & $x$ & $\checkmark$ & $x$ & $\checkmark$ \\
\hline $\mathrm{GD}_{1}$ & $\checkmark$ & $\checkmark$ & $\checkmark$ & $\checkmark$ & $\times$ & $\checkmark$ & $\checkmark$ & $\checkmark$ \\
\hline $\mathrm{GD}_{n}$ & $\checkmark$ & $\checkmark$ & $\checkmark$ & $\checkmark$ & $\checkmark$ & $\checkmark$ & $\checkmark$ & $\checkmark$ \\
\hline
\end{tabular}

The symbols $\checkmark$ and $\times$ show if the optimization produces a valid element or not, taking the vertex $\mathbf{x}_{i}$ as

free node

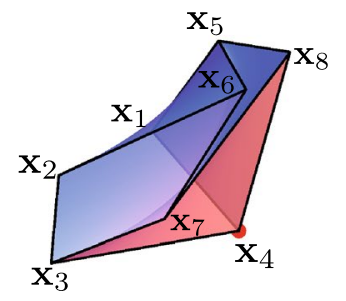

(d)

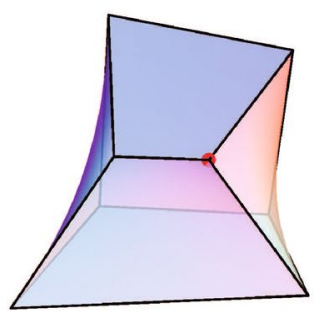

(b)

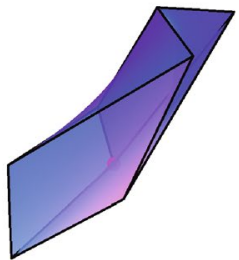

(e)

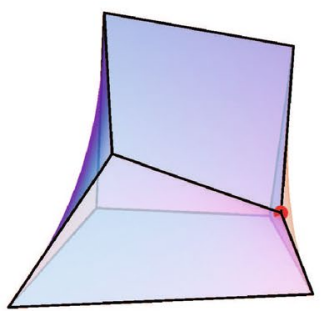

(c)

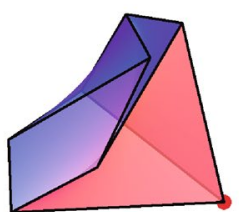

(f)

\subsection{Experiment 2}

In this experiment, we optimize a sufficiently large sample of highly distorted elements, collect statistical data, and compare the rate of success of each strategy.

The experiment is carried out on two types of samples. In the first one, we consider isolated hexahedral elements.
We generate $10^{5}$ highly distorted hexahedra, all of them valid. The elements are generated by placing the vertexes on random positions, with coordinates between 0 and 1 , and collecting only the cases when the hexahedron is valid. Generally, the elements generated by this procedure are deformed enough to test the untangling ability of the studied objective functions. 


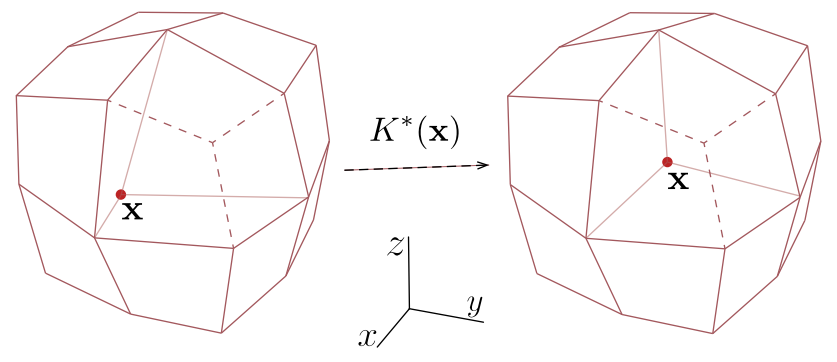

Fig. 8 Local mesh optimization

Table 2 Experiment 2 results

\begin{tabular}{lllll}
\hline Obj.function & $\mathrm{IE}_{s}$ & $\mathrm{IE} \%(\%)$ & $\mathrm{LM}_{s}$ & $\mathrm{LM} \%(\%)$ \\
\hline $\mathrm{TD}_{\alpha}\left(\mathrm{GD}_{0}\right)$ & 94,361 & 94.36 & 28,210 & 94.03 \\
$\mathrm{TD}_{\alpha \beta}$ & 87,965 & 87.96 & 17,620 & 58.73 \\
$\mathrm{TD}_{\alpha \gamma}$ & 66,852 & 66.85 & 11,669 & 38.90 \\
$\mathrm{TD}_{\alpha \kappa}$ & 92,155 & 92.16 & 25,279 & 84.26 \\
$\mathrm{TD}_{\alpha \beta \gamma \kappa}$ & 69,650 & 69.65 & 13,289 & 44.30 \\
$\mathrm{SC}$ & 91,947 & 91.95 & 25,853 & 86.18 \\
$\mathrm{GD}_{1}$ & 98,737 & 98.74 & 29,553 & 98.51 \\
$\mathrm{GD}_{n}$ & 99,968 & 99.97 & 29,944 & 99.81 \\
\hline
\end{tabular}

$\mathrm{IE}_{s}$ and $\mathrm{LM}_{s}$ represent the number of valid isolated elements and local meshes after optimizing. The sample sizes are $10^{5}$ for $\mathrm{IE}_{s}$ and $3 \times 10^{4}$ for $\mathrm{LM}_{s}$. The rates of success of each case are $\mathrm{IE}_{\%}\left(100 \mathrm{IE}_{s} / 10^{5}\right)$ and $\mathrm{LM}_{\%}\left(100 \mathrm{LM}_{s} / 3 \times 10^{4}\right)$

The second testing sample is composed by local meshes formed by eight hexahedral elements. Due to the low probability to generate a valid local mesh using random numbers, we have opted for a procedure driven by an objective function. In the appendix, we explain in detail the procedure used for the generation of highly distorted local meshes.

For each objective function, we count the number of isolated elements and local meshes that are valid after optimization. We have selected for the experiment only valid elements to be sure that the feasible region exists, i.e., the free node can be placed making the element valid. Otherwise, we could not be able to know whether a strategy fails to produce a valid element or this element just cannot be untangled. Note that it is irrelevant whether we start with a valid element or not, since the final position of the free node after optimization does not depend on its initial position. It is important to mention that some optimization strategies can tangle a valid element. Actually, none of the considered strategies is based on necessary and sufficient conditions for non-degeneracy of a hexahedron. Therefore, any of these strategies could tangle an initially valid element (specially, when the element is highly distorted).
For isolated elements, we take as free node $\mathbf{x}_{1}$, and for local meshes, the free node is the common vertex of the eight elements, see Fig. 8.

Table 2 shows the results of the experiment 2 . We start with some comments about the results of the first group of objective functions (TD). In this group, the objective function $\mathrm{TD}_{\alpha}$ reports the best results with the $94.36 \%$ rate of success for isolated elements and the $94.03 \%$ rate of success for local meshes. The remaining $\mathrm{TD}^{\prime} s$ involve a larger number of tetrahedra, and their untangling rates are lower.

When the terms corresponding to $\beta, \gamma$ or $\kappa$ tetrahedra are added, the objective function imposes sufficient but not necessary conditions for the validity of an element. These conditions are excessively restrictive and sometimes difficult to satisfy. Consequently, the optimization of this objective function may not place the free node within its feasible region. The objective function $\mathrm{SC}$ also obtains lower rates of success than $\mathrm{TD}_{\alpha}$. Note that both $\mathrm{TD}_{\alpha \beta \gamma \kappa}$ and $\mathrm{SC}$ impose sufficient conditions. However, the conditions used for SC strategy are less restrictive than the conditions of $\mathrm{TD}_{\alpha \beta \gamma \kappa}$, and thus, its success rate is higher than the rate of $\mathrm{TD}_{\alpha \beta \gamma \kappa}$.

The best optimization success rates are obtained with the $\mathrm{GD}^{\prime} s$ objective functions. In the case of $\mathrm{GD}_{1}, 98.74 \%$ of isolated elements and $98.51 \%$ of local meshes are valid after the optimization. With an adaptive distribution of the quadrature points, $\mathrm{GD}_{n}$, these rates are even better, with only 32 and 66 invalid isolated elements and local meshes, respectively. We suspect that the cases where $\mathrm{GD}_{n}$ fails are due to numerical problems in the optimization process. This can happen when the number of terms of the objective function is very large after performing several steps of adaptive subdivisions.

The computational cost of each strategy is mainly the cost of the minimization of the objective function. This minimization is more expensive as more terms (tetrahedra or integration points) are added to the objective function. $\mathrm{TD}_{\alpha}$ and $\mathrm{GD}_{0}$ are equivalent, see Remark 1. This strategy has only eight terms by element, and it is the most efficient strategy. Objective functions $\mathrm{TD}_{\alpha \beta}, \mathrm{TD}_{\alpha \gamma}, \mathrm{TD}_{\alpha \kappa}$, and $\mathrm{TD}_{\alpha \beta \gamma \kappa}$ have an approximate cost $4,6,2$, and 10 times higher than $\mathrm{TD}_{\alpha}$. The relative cost of $\mathrm{SC}$ and $\mathrm{GD}_{1}$ is 4 and 11 times the cost of $\mathrm{TD}_{\alpha}$. The most expensive strategy is $\mathrm{GD}_{n}$, but it strongly depends on the mesh where it is applied. When the mesh has relatively well-formed elements, the number of adaptive refinements required is lower than when the elements are highly distorted.

In practice, it is reasonable to optimize a mesh in two steps. Firstly, the objective function $\mathrm{TD}_{\alpha}\left(\mathrm{GD}_{0}\right)$ is applied. Then, the validity of the mesh is analyzed, and if needed, the mesh is optimized with $\mathrm{GD}_{n}$. 


\section{Conclusions and future research}

In this paper, we have analyzed the ability of several objective functions to optimize hexahedral elements. We have studied three groups of objective functions based on the distortion of some tetrahedra formed with the vertexes of the hexahedron (TD), sufficient conditions for the nondegeneracy of a hexahedron (SC), and a global distortion measure for the hexahedron (GD). We have performed two computational experiments to test the objective functions with highly distorted elements.

Some important conclusions can be extracted from the results of the experiments. The objective function $\mathrm{TD}_{\alpha}$ $\left(\mathrm{GD}_{0}\right)$ obtains good results with a lower computational cost than the rest of strategies. The addition of more tetrahedra to the objective function $\left(\mathrm{TD}_{\alpha \beta}, \mathrm{TD}_{\alpha \gamma}, \ldots\right)$ reports worse results and increases the computational cost. Objective function SC does not improve the success rates of $\mathrm{TD}_{\alpha}$, and its computational cost is higher, too. The best results are obtained with $\mathrm{GD}_{1}$ and, specially, with $\mathrm{GD}_{n}$, but with the disadvantage of increasing the computational cost with respect to $\mathrm{TD}_{\alpha}\left(\mathrm{GD}_{0}\right)$.

It is worth noting that the strategy $\mathrm{TD}_{\alpha}\left(\mathrm{GD}_{0}\right)$ obtains a quite high rate of success (94\%) for the sample of highly distorted meshes, which suggests even better results for moderately distorted meshes. So, in practice, it can be considered a reasonable choice since it succeeds in optimizing the great majority of real meshes. However, when dealing with extremely distorted meshes, a practical approach would be to optimize the mesh in two steps. Firstly, the optimization strategy $\mathrm{TD}_{\alpha}\left(\mathrm{GD}_{0}\right)$ is applied until reaching a stopping criterion, for example, that the nodes displacement is under a certain threshold. In the second step, the validity of the mesh is analyzed and, if there are some invalid elements, the mesh is optimized again with $\mathrm{GD}_{n}$.

An adaptive optimization strategy similar to $\mathrm{GD}_{n}$ can be especially useful for high-order hexahedral and tetrahedral meshes, which we plan in our future works.

Acknowledgments This work has been supported by the Spanish Government, "Secretaría de Estado de Universidades e Investigación," "Ministerio de Economía y Competitividad,", "Programa de FPU del Ministerio de Educación, Cultura y Deporte", "Programa de FPI propio de la ULPGC" and FEDER, grant contracts: CTM201455014-C3-3R and CTM2014-55014-C3-1-R.

\section{Appendix A. Bounds for Jacobian determinant}

To verify the validity of a physical element, we can minimize the Jacobian and check if its minimum value is negative or not. To reduce the computational cost, some bounds can be used to identify quickly some valid and invalid elements.

The Jacobian of the trilinear transformation is at most a degree two polynomial in each variable. The expansion of this polynomial in terms of Bernstein basis allows to find tight bounds for this polynomial. Let us consider the Jacobian

$J(\xi)=J(\xi, \eta, \zeta)=\sum_{i=0}^{l_{i}} \sum_{j=0}^{l_{j}} \sum_{k=0}^{l_{k}} a_{i j k} \xi^{i} \eta^{j} \zeta^{k}$.

In our case, $l_{i}, l_{j}, l_{k}=2$ and the total degree of the polynomial is 4 , i.e., $a_{i j k}=0$ if $i+j+k>4$. The expansion of $J(\xi)$ in terms of Bernstein basis is

$J(\boldsymbol{\xi})=\sum_{i=0}^{l_{i}} \sum_{j=0}^{l_{j}} \sum_{k=0}^{l_{k}} b_{i j k} B_{i}^{l_{i}}(\xi) B_{j}^{l_{j}}(\eta) B_{k}^{l_{k}}(\zeta)$

where $b_{i j k}$ are the control values, and

$B_{i}^{l}(\xi)=\left(\begin{array}{l}l \\ i\end{array}\right) \frac{(\xi+1)^{i}(1-\xi)^{l-i}}{2^{l}}$

is the $i$ th Bernstein polynomial of degree $l$ in the interval $[-1,1]$.

An explicit expression for the control values $b_{i j k}$ can be found in [15]. Due to the properties of the Bernstein functions, i.e., positivity and partition of unity, it can be shown that the control values bound the Jacobian

$\min _{i, j, k} b_{i j k} \leq J(\xi) \leq \max _{i, j, k} b_{i j k}$,

where $0 \leq i \leq l_{i}, 0 \leq j \leq l_{j}$, and $0 \leq k \leq l_{k}$.

The minimum of $b_{i j k}$ sets a tight lower bound for $J(\xi)$, so the condition min $b_{i j k}>0$ determines the validity of an element with a low computational cost. Only in a few ambiguous cases, when $\min b_{i j k} \leq 0$ and $\max b_{i j k}>0$, we have to minimize $J(\xi)$ to check if the element is valid. More accurate bounds can be found by recursively splitting the initial interval in smaller ones [16, 17]. This option is specially appropriate for high degree polynomials in which the minimization is computationally impractical.

\section{Appendix B. Random local meshes generation}

Isolated hexahedra for the first sample of the experiment 2 is constructed by assigning a random value from the interval $[0,1]$ to the coordinates of the eight vertexes of the element. Then, the validity of the hexahedron is checked, and only valid ones (positive Jacobian) are selected to participate in the experiment. The probability to generate a 
valid random hexahedron is approximately $1 / 1000$, i.e., one valid element is generated per 1000 randomly generated elements. Due to the low computational cost to generate a random element, it is feasible to obtain a sufficiently big sample for the experiment.

For the second sample of the experiment 2, we need a set of random local meshes. As we are dealing with local meshes formed by eight elements, we have to generate randomly 26 of the 27 nodes that compose the local mesh (we can fix the free node). We need to select for the experiment only valid meshes, i.e., each element of the local mesh should be valid. However, during random generation of the nodes' coordinates, the probability to generate a valid local mesh is very low. In fact, we could not generate any valid local mesh using this procedure. Therefore, another approach was used to generate a sample of highly distorted local meshes. The procedure is as follows. We take a local mesh composed by eight ideal elements, and deform it by moving 26 nodes of the local mesh. The deformation is performed by means of an optimization process where the nodes $\left\{\mathbf{p}_{j}\right\}_{j=1, \ldots, 26}$ are relocated to achieve a predefined distortion at certain points of each element of the local mesh. First, we select a set of points where we intend to impose the distortion. For example, we are going to impose the predefined distortion at the vertexes of each element, so there are $8 \times 8$ points. Let us denote these points as $\left\{\mathbf{x}_{k, i}\right\}_{k, i=1, \ldots, 8}$, where the index $k$ is for the elements numeration, and the index $i$ is for the nodes numeration of the $k$-th element. Next, we assign to each of these points a distortion value $\bar{\eta}_{k, i}=1 / \bar{q}_{k, i}$, where the quality $\bar{q}_{k, i}$ is a randomly generated number from $[\epsilon, 1]$. Then, the goal is to deform the initial mesh so that for each element $\Omega_{k}$, its trilinear mapping $\mathbf{x}^{k}(\xi)$ has the predefined distortion values at the corners $\left\{\mathbf{x}_{k, i}\right\}_{i=1, \ldots, 8}$ of the element, that is

$\eta_{k, i}^{*}=\frac{\left\|S_{k, i}\right\|^{2}}{3 h\left(\sigma\left(S_{k, i}\right)\right)^{2 / 3}}=\bar{\eta}_{k, i}$,

where $S_{k, i}$ is the Jacobian matrix of the mapping $\mathbf{x}^{k}(\xi)$ evaluated in the $i$-th node of the element $\Omega_{k}$. For that, the 26 nodes are relocated by minimizing the following objective function

$$
\begin{aligned}
F\left(\mathbf{p}_{1}, \mathbf{p}_{2}, \ldots, \mathbf{p}_{26}\right) & =\sum_{k=1}^{8} \sum_{i=1}^{8}\left(\eta_{k, i}^{*}-\bar{\eta}_{k, i}\right)^{2} \\
& =\sum_{k=1}^{8} \sum_{i=1}^{8}\left(\frac{\left\|S_{k, i}\right\|^{2}}{3 h\left(\sigma\left(S_{k, i}\right)\right)^{2 / 3}}-\bar{\eta}_{k, i}\right)^{2} .
\end{aligned}
$$

As a result of this minimization, we obtain the new position of the nodes $\left\{\mathbf{p}_{j}\right\}_{j=1, \ldots, 26}$ that provide a highly distorted local mesh. These local meshes are not necessarily valid, so analogously to the first sample, the validity is checked, and only valid local meshes are selected for the experiment. It is worth noting that, in general, it is not possible to construct a hexahedron with predefined quality at certain set of points, because the required qualities may not be compatible. However, this procedure provides a good method for generating arbitrary highly distorted local meshes.

\section{References}

1. Benzley S, Steven E, Perry E, Merkley K, Clark B, Sjaardema G (1995) A comparison of all-hexahedral and all-tetrahedral finite element meshes for elastic and elasto-plastic analysis. In: Proceedings 4th international meshing roundtable, Sandia National Laboratories, pp 179-191

2. Cifuentes AO, Kalbar A (1992) A performance study of tetrahedral and hexahedral elements in 3D finite element structural analysis. Finite Elem Anal Des 12(3-4):313-318

3. Weingarten V (1994) The controversy over hex or tet meshing. Mach Des 66(8):74-76

4. Knupp PM (2001) Algebraic mesh quality metrics. SIAM J Sci Comput 23:193-218

5. Knupp PM (2003) A method for hexahedral mesh shape optimization. Int J Numer Methods Eng 58(2):319-332

6. Wilson TJ, Sarrate J, Roca X, Montenegro R, Escobar JM (2012) Untangling and smoothing of quadrilateral and hexahedral meshes. In: Proceedings of the eighth international conference on engineering computational technology

7. Ushakova OV (2011) Nondegeneracy tests for hexahedral cells. Comput Methods Appl Mech Eng 200(17-20):1649-1658

8. Gargallo-P eiró A (2014) Generation of curved meshes for highorder unstructured methods. Ph.D. thesis, Universitat Politècnica de Catalunya

9. Knupp PM (1990) On the invertibility of the isoparametric map. Comput Methods Appl Mech Eng 78(3):313-329

10. Escobar JM, Rodríguez E, Montenegro R, Montero G, GonzálezYuste JM (2003) Simultaneous untangling and smoothing of tetrahedral meshes. Comput Methods Appl Mech Eng 192:2775-2787

11. Escobar JM, Rodríguez E, Montenegro R, Montero G, GonzálezYuste JM (2003) SUS code: simultaneous mesh untangling and smoothing code, Technote, http://www.dca.iusiani.ulpgc.es/ SUScode/TechNote.pdf

12. Dennis J, Schnabel R (1983) Numerical methods for unconstrained optimization and nonlinear equations, classics in applied mathematics, society for industrial and applied mathematics, Englewood Cliffs, New Jersey. http://books.google.es/ books?id=RtxcWd0eBD0C

13. Knupp PM (2003) Algebraic mesh quality metrics for unstructured initial meshes. Finite Elem Anal Des 39:217-241

14. Knabner P, Korotov S, Summ G (2003) Conditions for the invertibility of the isoparametric mapping for hexahedral finite elements. Finite Eleme Anal Des 40:159-172

15. Garloff J, Jansson C, Smith AP (2003) Lower bound functions for polynomials. Comput Appl Math 157(1):207-225

16. Johnen A, Remacle JF, Geuzaine C (2014) Geometrical validity of high-order triangular finite elements. Eng Comput 30(3):375-382

17. Geuzaine C, Johnen A, Lambrechts L, Remacle JF, Toulorge T (2015) The generation of valid curvilinear meshes. In: N Kroll, C Hirsch, F Bassi, C Johnston, K Hillewaert (eds) IDIHOM: industrialization of high-order methods-a top-down approach, vol 128, Springer International Publishing, Berlin, pp 15-39 